\title{
De la IAP a las Metodologías Sociopráxicas
}

\section{From IAP to Sociopraxics Methodologies}

\author{
Manuel Montañés Serrano* y Pedro Martín Gutiérrez**
}

Fecha de recepción: 27-04-2017 - Fecha de aceptación: 16-09-2017

Hábitat y Sociedad (ISSN 2173-125X), n. ${ }^{\circ}$ 10, noviembre de 2017, pp. 35-52.

http://dx.doi.org/10.12795/HabitatySociedad.2017.i10.03

\begin{abstract}
After seventeen editions, due to budgetary policies, it was not possible to give a postgraduate course at the Universidad Complutense de Madrid, which would have been pioneering in Spain, offering training and implementation of Participatory Methodologies. The article claims against the poor institutional support provided to this type of methodology which, however, are applied with more or less success in extra-academic fields around the world, this article aims at reporting on the epistemological, theoretical / methodological and technological development as experienced according to the IAP (participatory action research, which provided the first reference to the course), through to the sociopraxic methodologies elaborated by all those involved in these seventeen years through active participation.
\end{abstract}

\section{Key words}

Citizen participation; Constructivism; Participatory Action Research; Participatory methodologies; Sociopraxis

\section{Resumen}

Tras diecisiete ediciones, debido a las políticas presupuestarias, no se ha podido impartir en la Universidad Complutense de Madrid un curso de posgrado pionero en España en la formación y aplicación de Metodologías Participativas. Este artículo, además de servir de denuncia del escaso apoyo institucional prestado a este tipo de metodología que, sin embargo, son prolijamente aplicadas, con más o menos acierto, en ámbitos extraacadémicos en todo el mundo, se propone dar cuenta del desarrollo epistemológico, teórico/metodológico y tecnológico experimentando desde las IAP (que inicialmente sirvieron de referencia al curso) a las metodologías sociopráxicas elaboradas participativamente por todas las personas implicadas en estos diecisiete años.

\section{Pallabras clave}

Constructivismo; Investigación Acción Participativa; Metodologías participativas; Participación ciudadana; Sociopraxis

\footnotetext{
* Doctor y licenciado en Ciencias Políticas y Sociología; profesor de la Universidad de Valladolid. Universidad de Valladolid (Campus de Segovia). Plaza de la Universidad, 1, CP 40005, Segovia. C.e.: investigacionparticipada@gmail.com.

** Doctor y licenciado en Sociología; profesor de la Universidad de Valladolid. C.e.: pmg@soc.uva.es.
} 


\section{Presentación ${ }^{1}$}

El curso Especialista Universitario en Investigación Participativa y Gestión Local, Título Propio de la Universidad Complutense de Madrid, se imparte por primera vez en el año académico 1995-1996, dirigido por Tomás R. Villasante, profesor de Sociología de la UCM, y por un equipo de profesores de manera colegiada, siendo dos de sus componentes los redactores del presente artículo. En 2001-2002 pasó a denominarse Máster en Investigación, Gestión y Desarrollo Local y en una propuesta posterior se sustituiría el término Gestión por el de Metodologías Participativas. Estos cambios en la denominación del curso van dando cuenta de las transformaciones y la orientación que va tomando este.

Todos los proyectos que se ponen en marcha tienen unos antecedentes. Los de este curso es posible rastrearlos en la actividad militante, política y ciudadana de sus promotores en tiempos del final de la Dictadura y el inicio del periodo democrático, cuando la participación se hacía "porque era lo que había que hacer" y el hacer ciudad era sinónimo de hacer ciudadanía activa y democrática. Aquí se produjo, en gran medida, el aprendizaje práctico que posteriormente se teorizaría en profundidad para de nuevo volver a aplicarlo en la acción. También desde su comienzo se vinculó si no directa y exclusivamente a la universidad, sí a profesores y profesionales que no se desprendieron de su nexo universitario ni de sus nexos vecinales.

El objetivo principal de estos cursos, tal como lo recoge uno de los primeros convenios firmados con ayuntamientos de la Comunidad de Madrid, es el de "contribuir a formar especialistas en técnicas de investigación sociológica, concretando este campo en las técnicas participativas de personas, entidades, asociaciones o grupos en la vida local y los intereses públicos municipales" (Convenio UCM y Ayuntamiento de Fuenlabrada, 1995, p. 1). El anexo de este convenio recogía el propósito del trabajo a desarrollar: "contar con el mayor número de datos y propuestas que le permitan seguir avanzando en su relación con entidades, grupos y colectivos existentes en la ciudad para mejorar la planificación de servicios y la implicación ciudadana" (ob. cit.).

Este tipo de convenios con las administraciones públicas y entidades sociales no solo aportaba la demanda para realizar los trabajos prácticos de investigación, sino también los recursos económicos con los que pagar los honorarios por las clases, tutorías, contratos para la coordinación académica y gestión administrativa - la dirección siempre se realizó gratuitamente-, las becas para quienes justificaban dificultades económicas, además de ingresar un porcentaje —en el último curso el $25 \%$, con propuesta del $35 \%$ para el siguiente- a los servicios centrales de la universidad. Este curso se ha financiado con las tasas académicas pagadas por el alumnado (siempre en el rango más bajo de los cursos de posgrado en la universidad española) y por los recursos que los convenios aportaban. Esta modalidad ha contribuido a que los avatares de cada edición influyeran en la mayor o menor solvencia, hasta llegar a su inviabilidad económica.

La formación, como hemos dicho, con un fuerte componente práctico, se pretendía que fuera complementaria de la académica, al tiempo que encajase con alguna de las actividades técnicas que se requerían en las administraciones públicas y las organizaciones y movimientos sociales. Era el momento en que se demandaba un buen número de profesionales para la gestión de las áreas municipales de participación

1 Este artículo tiene su base en la popañol de Sociología, celebrado en Gijón el 30 de junio y el 1 y 2 de julio de 2016. 
o de Desarrollo Local (Montañés et al., 1999), y también en la gestión asociativa de todo tipo.

A pesar de los cambios que este curso ha experimentado en su trayectoria, no ha variado el principio según el cual el eje vertebrador de esta formación debía ser el trabajo de investigación práctica. Con los conocimientos básicos sobre metodologías participativas, los equipos de trabajo llegaban a campo y empezaban a conocer materialmente la problemática que se debía abordar. De este modo, las clases sobre el módulo troncal metodológico eran reflexiones prácticas, dado que las dudas y preguntas que planteaba el trabajo siempre solían ser más abundantes e iban por delante de la explicación o la lectura teórica. Esta presión del trabajo era compensada con la presencia de un profesional, que ya había pasado por el curso, y que tutorizaba el trabajo de los equipos.

Si bien la reflexión, a partir de la práctica, no solo tenía lugar en los equipos de trabajo de cada promoción, sino que también era compartida por el equipo de dirección y coordinación del curso. Tal como tenemos a gala postular, nadie sale intacto de estos procesos práxicos, los cuales nos transforman en el acto de transformar la realidad sobre la que intervenimos. En esta línea, cada año se han realizado unas Jornadas de Reflexión. Al principio, con un formato más clásico, con la intervención de ponentes de renombre (en dos ocasiones se han realizado bajo el marchamo de "Seminario Internacional Complutense"), pero, buscando la coherencia, han pasado a ser autogestionadas por un grupo promotor en un proceso participativo, al que se suman otras personas, colectivos, organizaciones, etc.

Es este modelo teórico-metodológico al que en un principio nos adscribimos, con la denominación ya conocida y genérica de Investigación-Acción Participativa (IAP). Si bien, la constante inquietud reflexiva ha hecho que se reelaborase, una y otra vez, desde las técnicas a la base epistemológica de la propuesta con la que trabajamos, a la que denominamos metodologías de investigación sociopráxica.

Este proceso, además de producir más de 70 investigaciones, ha propiciado la reflexión epistemológica, la producción teórico-metodológica, la elaboración y construcción tecnológica, la provocación (porque se ha propiciado el dar voz) de debates entre diferentes grupos de investigación y acción y la creación de redes de relaciones que se han concretado en grupos de trabajo profesional, asociativo o investigador, tanto en el Estado español como en Latinoamérica. También ha sido amplia la producción académica, con un número considerable de tesis doctorales, proyectos de investigación y la introducción de estas metodologías en numerosas universidades, tanto de un lado como de otro del Atlántico.

Pero estas actividades colectivas tienen también una impronta profesional, se ha de tener en cuenta que por este curso universitario han pasado más de 300 alumnos y alumnas, además de los que se han formado específicamente en asesorías e investigaciones llevadas a cabo mediante convenios Artículo 83 (Transferencia de Resultados de Investigación) y similares. Se ha podido constatar, mediante una investigación realizada a propósito de las experiencias de Presupuestos Participativos en España (Ganuza y Francés, 2012), que la formación para asesorar a los cargos políticos y capacitar a los técnicos responsables de las áreas de participación de las Administraciones Locales, se ha llevado a cabo, en buena medida, en unos pocos cursos universitarios, de los cuales al que aquí se está haciendo referencia ha sido pionero y generador de otros. 
El curso de Posgraduado fue replicado a finales de los años noventa en la Universidad Autónoma de Barcelona, en la Universidad Pablo de Olavide de Sevilla y en la Universidad del País Vasco [...]. Estos cursos tendrán una relevancia notable en los orígenes de los presupuestos participativos, pues más de la mitad de las experiencias de presupuestos participativos (hasta el año 2007) contrataron a personas formadas directamente en ellos, en algunos casos incluso se contrató un asesoramiento por parte del mismo curso (Cabezas de San Juan o Figaró), es decir, los alumnos del curso universitario hacían sus prácticas en el municipio, en estos casos, sobre el presupuesto participativo (Ganuza y Francés, 2012, p. 97).

También han sido muy numerosos los técnicos y especialistas que se han formado, para posteriormente integrarse profesionalmente en el campo del desarrollo local, a los que se les encargaba un trabajo -en ocasiones indefinido, inespecífico, con una enorme variabilidad-que requería una formación con contenidos estratégicos y creativos, aunque debiendo hacerse compatible con la pertinente capacidad operativa e instrumental. En un estudio que se realizó para FOREM, en sus conclusiones se afirmaba que era necesaria una "formación 'certificada', avalada por entidades u organismos de prestigio, susceptible de ser apreciada en convocatorias de empleo o de promoción laboral" (Montañés et al., 1999, p. 161), pudiéndose comprobar que este curso era uno de los pocos reconocidos en las convocatorias oficiales para estos puestos de trabajo.

La trayectoria de esta actividad formativa, aunque no demasiado prolongada, parece que no ha sido desaprovechada.

\section{Origen y desarrollo de las Metodologías Participativas}

La propuesta metodológica que hemos desarrollado no solo difiere del modo clásico de proceder, sino también de las clásicas metodologías participativas.

Fue Kurt Lewin quien en 1946 acuñó el término action-research, tomando como referencia la psicosociología americana del propio Kurt Lewin y de Carl Rogers, principalmente, que había demostrado el eficaz papel desempeñado por los grupos e individuos en las terapias psicosociales (Faber et al., 2001). La action-research proponía no separar la planificación de la aplicación de las propuestas con las que intervenir socialmente; quedando, de esta manera, rota la clásica distinción entre productores de conocimiento (científicos sociales/planificadores) y quienes se encargan de la aplicación de los mismos (trabajadores sociales/ejecutores). De acuerdo con Kemmis y McTaggart (1987), la $a c$ tion-research pone el centro de gravedad en el grupo. El grupo, a partir de un deseo de cambio, propone la temática, diagnóstica, propone el plan de acción y posteriormente evalúa los resultados. Este enfoque supuso un salto cualitativo respecto al tradicional modo de proceder de los profesionales del trabajo social, pero, como se ha dicho, el acento se sitúa en el equipo y no tanto en quienes se benefician o padecen las actuaciones que este equipo pone en marcha.

Si en inglés la acción se enuncia en primer lugar, en francés la investigación es la que aparece primero (recherche-action). Charles Delorme (1982) y Henri Desroche (1979), entre otros, son quienes emplean este término. Este enfoque persigue explicar la situación y el proceso, aplicar la acción e implicar a sus actores, que emprenden sus 
acciones teniendo en cuenta la información proporcionada por la investigación realizada. Es una forma de autogestionar tanto la producción de conocimiento como las acciones a desarrollar (Meister y Retour, 1980).

En Latinoamérica, el término utilizado es el de Investigación Participativa. Fals Borda (1993), Schutter (1985), Vio Grossi (1981), Molano (1978), Oquist (1978), Rojas (1978), Briones (1978), Núñez et al. (1990) o Sanguinetti (1981) son algunos de los profesionales latinoamericanos que se adscriben, aunque con sus correspondientes matices, en esta perspectiva metodológica. ${ }^{2}$ Este enfoque se enmarca en un compromiso militante en defensa de los más desfavorecidos. Tiene su influencia en la pedagogía liberadora de Paulo Freire (1970) y en la concepción marxista del conocimiento. No se concibe una ciencia exenta de valores y ajena a los procesos sociales, sino todo lo contrario, se considera que la ciencia está al servicio de las clases que la producen, de ahí que abogue por articular mecanismos para que las clases más desfavorecidas produzcan sus saberes, y así contrarrestar el de los que están al servicio de las clases dominantes.

De acuerdo con Orlando Fals Borda, el método de la Investigación Participada se estructura en cuatro pasos: 1) la investigación colectiva o de grupos; 2) la recuperación histórica; 3) la valoración y utilización de los elementos de la cultura popular; y 4) la comunicación multivocal de los trabajos. Por investigación colectiva se entiende la recolección de datos mediante reuniones, sociodramas, asambleas públicas, comités y demás actividades colectivas. En el apartado dedicado a la recuperación crítica de la historia se propone, a través de la memoria colectiva, recuperar aquellos elementos del pasado que han demostrado ser de utilidad en la defensa de los intereses de las clases explotadas, los cuales pueden ser utilizados en las luchas del presente para lograr un momento de la "concientización". En cuanto a la valoración y aplicación de la cultura popular se propone recuperar prácticas culturales ignoradas por las clases dominantes, como son la música, las artes, el drama, los deportes, las creencias, los mitos, los cuenteros, etc. Todo ello dinamizado y difundido a través de canales de comunicación comunitarios de carácter alternativo a los medios dominantes (Fals Borda, 1993).

En España, es Paloma López de Ceballos quien populariza el término IAP (López de Ceballos, 1987), que toma su nombre de dos escuelas: la investigación participativa y la investigación-acción. López de Ceballos sitúa en la base del proceso a los GIAP (los grupos de investigación-acción participativa). A estos les asigna el peso fundamental de la investigación y formulación de propuestas.

Un planteamiento similar, sustentado en un equipo de personas interesadas en una determinada problemática, fue llevado a cabo por el antropólogo estadounidense Greenwond en un pueblo de Castilla la Mancha. En este caso, la investigación participada, consistió en:

un cursillo breve sobre investigación-acción a un grupo grande de enseñantes del pueblo y luego colaborar con ellos en cuanto grupo organizador de un proceso comunitario. También se les convocó para desarrollar una "búsqueda" (search conference) [según su autor: "reunión de los dueños del problema durante dos días para replantearse su futuro"], un proceso de planificación estratégica comunitaria, en el cual participaron 45 miembros de la comunidad [creándose] varios equipos que empezaron a trabajar sobre problemas específicos (Greenwood, 2000, p. 45).
2 En 1977 tuvo lugar en la ciudad colombiana de Cartagena de Indias el "Simposio Mundial sobre Investigación-Acción y Análisis Científico", impulsado por Orlando Fals Borda y otros muchos activistas e investigadores, para poner en valor los procedimientos de investigación-acción que se estaban practicando por tierras americanas; 20 años después, en 1997, se realizó un encuentro similar en la misma ciudad, revisando los logros y retos que la IAP se planteaba. En fechas recientes, y tras otras dos décadas (junio de 2017), de nuevo Cartagena ha acogido a una amplia representación de las más diversas tendencias, estilos y prácticas de las metodologías participativas en el encuentro sobre "Participación y Democratización del Conocimiento: Nuevas Convergencias para la Reconciliación", organizado por la Universidad Nacional de Colombia y ARNA (Action Research Network of the Americas); en el mismo se ha rendido homenaje a sus precursores y, asimismo, se han puesto de manifiesto tanto las convergencias como las discrepancias generadoras, así como los desbordes creativos de estas. 
Este mismo autor había dirigido mucho antes (en 1985) una investigación participada en el ámbito de una factoría, concretamente en FAGOR, empresa perteneciente al grupo de Cooperativas de Mondragón (Greenwood y González, 1989).

Hay también antropólogos españoles, como Ángel Montes del Castillo (1989), que realizan investigaciones participadas fuera de nuestras fronteras. En Pucará, una comunidad campesina mestiza de los Andes ecuatorianos, las metodologías participativas aplicadas a un estudio antropológico contribuyeron a una mayor y mejor recolección de datos. Ello fue posible gracias a la Asamblea Participativa en la que el grupo social investigado participaba activamente en esta tarea. Montes subraya cómo este modo de proceder rompía, en gran medida, la dicotomía sujeto/objeto, o si se prefiere observador/observado (antropólogo investigador/grupo social investigado).

Como se puede apreciar, todos los enfoques, con más o menos énfasis, tienen cierta dosis de compromiso social: desde una postura más militante a otra de implicación psicoafectiva con el grupo con el que se trabaja. Pero, sin duda, el aspecto más relevante y que unifica a todos los enfoques es el de propiciar que un grupo no académico sea sujeto de la investigación, esto es, que participe en la producción de conocimiento, en la formulación de las acciones, así como en las acciones que hayan programado.

En unos casos se trata de que un grupo analice, programe y evalúe las acciones a desarrollar en su trabajo con otras personas; en otros que un grupo, más o menos amplio, estudie, planifique y evalué las acciones cuyos destinatarios son ellos mismos y así poder contrarrestar el saber dominante; en un tercero que un grupo, más o menos activo, reciba la pertinente formación para abordar procesos participativos en el ámbito donde residen o trabajan; y en un cuarto que el grupo aporte de manera participada la información y conocimientos sobre la realidad estudiada. En todos los enfoques hay un otro que, dentro de su posible heterogeneidad interna, constituye una única realidad grupal, esté o no formalmente constituido (léase profesionales del trabajo social, empleados de un centro de trabajo, miembros de una asociación, grupos juveniles, de mujeres, miembros de una cooperativa, grupo étnico cultural, etc., pongamos por caso). Sean unos u otros casos, la otre$d a d$ se presenta al relacionar el sujeto investigador (el profesional de las ciencias sociales) y los objeto-sujetos-investigados, abogándose porque estos últimos (los objetos-sujetos) dejen de ser objetos para convertirse en sujetos de la investigación del mismo modo que lo es el sujeto-profesional de la investigación. En cambio, en la estrategia metodológica que aquí se propone, la otredad va más allá del sujeto investigador-grupo social investigado. De acuerdo con este planteamiento, se considera que todos somos otros de otros. No habiendo una sola alteridad sino tantas como se sea capaz de construir. En las ciudades no hay una sola otredad. Ni el saber no académico es solo uno. Por tanto, no se aboga por trabajar al servicio de un grupo, ni tampoco para que un grupo autogestione su conocimiento, ni por instruir a un grupo determinado para que estudie y formule propuestas de actuación - todas ellas actividades plausibles, dicho sea de paso-, sino que se propone emprender una estrategia para que todas las realidades grupales, observando la observación de todas las realidades grupales presentes en una localidad, puedan formular problemas y aportar soluciones a los mismos. 


\section{Justificación epistemológica}

Dado que el cerebro no tiene ninguna apertura al exterior, no es posible conocer la realidad externa. El ser humano no puede conocer nada del exterior, sino es infiriéndole su sentido. No vivimos en el vacío, fuera de nosotros hay un ambiente, pero ese ambiente, y quienes habitan en el mismo, cobran significado social al inferirles nuestro particular sentido. Fuera de nosotros, no hay conceptos, pero tampoco hay árboles ni casas ni montañas, tanto los conceptos como los árboles, las casas y las montañas cobran existencia al dotar de sentido al ambiente energético en el que habitamos. Toda realidad es construida. Si bien, conviene aclarar que esta construcción no responde a la introspección caprichosa de cada cual (como desde un constructivismo abstracto pudiera defenderse). El ser humano, en tanto que sistema organizacionalmente e informacionalmente cerrado y energéticamente abierto, al analizar y valorar las perturbaciones (ya sean de origen internos o externo) procede a su clausura organizacional (Pask, 1981), construyendo la realidad a la par que se autocrea, pero esta autonomía se encuentra condicionada, como así lo entienden Maturana y Varela, por el acoplamiento estructural con otros organismos (Maturana y Varela, 1990), por lo que la supervivencia nos exige que construyamos realidades compatibles con las de otros seres humanos con los que nos acoplamos estructuralmente. Mal nos iría si con todos y cada uno de los seres humanos con los que nos vemos obligados a acoplarnos para atender nuestras necesidades no compatibilizáramos realidad alguna.

Ahora bien, conviene aclarar que compatibilizar no es lo mismo que compartir. Compartir implica la existencia de una externa realidad universal que engloba a, o de la que participan diversos sujetos. Compatibilizar significa que la realidad de uno encaja en la realidad de otro u otros. Como señala von Glasersfeld:

hablar de significados compartidos es un sinsentido puro [...]: no he construido el lenguaje como no he construido esta mesa, pero me he adaptado a la mesa no atravesándola. Me he adaptado al lenguaje que existe construyendo mis significados de manera tal que encajen en mayor o menor medida con los significados de los otros. Pero "encaje" no es equivalencia. "Compatible" no quiere decir "igual", simplemente significa que no causa problema (von Glasersfeld, 1994, p. 138).

Si no hay una realidad para ser observada, se ha de admitir que no hay verdad por descubrir. "La verdad — como dice Jesús Ibáñez- no es una pieza a cobrar, sino un universo a ensanchar" (Ibáñez, 1990, p. 7). O, como afirma, Eduardo Galeano, "la verdad está en el viaje, no en el puerto. No hay más verdad que la búsqueda de la verdad" (Galeano, 1998, p. 336). O, como sostiene Eduardo Crespo, "la verdad no es un estado de la realidad que se descubre, sino un tipo de afirmación sobre la misma que se discute y pone a prueba: es un problema práctico" (Crespo, 2003, p. 23). En consecuencia, la legitimidad científica no puede recaer ni en la teoría de la que se parte, ni en la valoración de unos imposibles empíricos resultados externos observables por todo el mundo, sino que ha de descansar en el modo de proceder. En sí el procedimiento seguido para producir conocimiento y propuestas de actuación participan o no los diversos grupos/sistemas observadores que se ven o pueden verse afectados por las acciones perceptivomotoras que tienen y tendrán lugar, a tenor del producto resultante de la realidad construida. 
Si Descartes (1961) consideraba al sujeto un elemento que era preciso anular o, al menos, amortiguar lo más posible por ser generador de ruido y distorsionador de la producción de conocimiento - con este fin separa al sujeto pensante (ego cogitans: el mundo de lo pensante) de las cosas materiales (res extensa: el mundo de los objetos físicos dotados de extensión, figura y movimientos) - , desde el paradigma que aquí se defiende, la participación del sujeto, como se ha argumentado, resulta imprescindible.

\section{Fundamentación teórica}

El modo clásico de proceder se sustenta en un programa, el sociopráxico, en una estrategia.

La palabra estrategia se opone a la palabra programa. Para las secuencias que se sitúan en un ambiente estable, conviene utilizar programas. El programa no obliga a estar vigilante. No obliga a innovar [...] una estrategia, por el contrario, se determina teniendo en cuenta una situación aleatoria, elementos adversos, e, inclusive, adversarios, y está destinada a modificarse en función de las informaciones provistas durante el proceso, puede tener así una gran plasticidad (Morin, 1995, pp. 115-127).

La sociología clásica, una vez construida la estructura, como si de un edificio se tratara, comienza a completarla, a cerrar los interiores. La investigación sociopráxica, por su parte, se ha de encontrar en permanente reelaboración.

Esta permanente reelaboración facilita encontrar respuestas a preguntas realizadas y también encontrarnos con preguntas y respuestas no formuladas.

En la investigación clásica el paradigma científico que la sustenta solo permite formular problemas cuya solución se encuentra implícitamente incluida. Desde la perspectiva sociopráxica, el quehacer sociológico ha de ayudar tanto a resolver problemas como a plantear problemáticas cuyas soluciones no se hallan implícitas. La investigación sociopráxica carece de bordes que la limiten.

Al no tener límite, la producción de conocimiento y propuestas de actuación de manera participada es imposible que sea lineal, esta ha de ser, asumiendo los principios de la lógica compleja, dialógica, hologramática y recursiva (Morin, 1995). Es decir, se ha de proceder de tal modo que quienes sostienen diferentes lógicas (que no son más que ideologías de uno o unos) conversen en la construcción de propuestas. Quedando incluida cada una de las partes del proceso en el todo y en cada una de las partes del proceso. Siendo cada parte causa y efecto. Dicho de otro modo,

El devenir del proceso se verá condicionado por cada etapa, las cuales experimentarán modificaciones como resultado de la dialogización que tenga lugar en las conversaciones a tal efecto realizadas, dando lugar a la programación de otras (conversaciones) en donde se establecerá el modo de proceder de acuerdo con el proceso que ha hecho posible que tengan lugar las conversaciones en donde se ha previsto el modo de proceder. Dibujando el proceso una trayectoria espiral. Las idas y venidas producirán círculos inacabados en niveles superiores (Montañés, 2003, p. 118). 
El diseño del modo de proceder queda abierto, pero ello no ha de significar que la producción de conocimiento y propuestas de actuación de manera participada quede a expensas de la improvisación permanente. Se ha de orientar, formular, sistematizar y organizar el modo de proceder.

A partir de un requerimiento inicial, el equipo técnico ha de elaborar un documento de trabajo en donde se proponga una demanda explícita que deberá someter a negociación. Ha de diseñar un proyecto que propicie la elaboración de un proceso, siendo, como se ha dicho antes, las conversaciones las que irán redefiniendo el proyecto de la participación conversacional.

Todo es producto y productor de todo. Se ha de diseñar un proceso con el que lograr que todo y todas y todos tengan la posibilidad de incidir en todo y en todos y todas. Ello requiere que todos los grupos/ sistemas observadores puedan inferir sentido a lo observado por todos los grupos/sistemas observadores (incluida la observación del sujeto investigador).

En la investigación clásica, el científico social ejerce de kantiano sujeto transcendente observador del mundo; en otras, llamadas participadas, también lo puede seguir ejerciendo, ocultando ese papel haciendo partícipe a la población en cuestiones técnica o/y metodológicas. Para que realmente desaparezca esta figura, las múltiples realidades grupales han de poder observar la observación del conjunto de realidades grupales. Todas han de poder emitir energía y han de poder inferir sentido a la energía emitida. Ello implica sustituir la lógica por la dialógica, en donde se ponga en evidencia la pugna que mantienen entre sí todas las relaciones ideológicas por convertirse en relaciones lógicas. Lo que nos lleva a sustituir tanto el método deductivo como el inductivo por la abducción. En la abducción no se va de lo general a lo particular (deducción), ni de lo particular a lo general (inducción), sino que se agregan discursos con los que proyectar nuevos escenarios posibles. Todo ello insertado en un proceso transductivo que articula lo nuevo en lo conocido, pues nunca se parte de cero.

En consecuencia, la separación entre doxa (conocimiento del vulgo) y episteme (conocimiento científico) que, desde Platón, la producción de conocimiento ha establecido, según la perspectiva de la participación conversacional, que aquí se defiende, queda suprimida. En sus efectos, tan real es la realidad del investigador, con la que explica el devenir de los seres humanos, como la realidad de los sujetos investigados. Piénsese en la creencia en Dios. Carece de sentido mostrar la existencia o no de Dios. En cambio, sí resulta pertinente, a todas luces, prestar atención tanto a por qué y para qué se cree en Dios como a las consecuencias que se derivan de la creencia en la existencia de Dios.

La investigación sociopráxica, al objeto de aunar episteme y doxa, ha de articular el enfoque emic y etic desde una perspectiva proyectiva.

El par emic/etic fue acuñado por Pike, en la década de los cincuenta del anterior siglo, tomando como referencia dos disciplinas lingüísticas: la fonología (en inglés phomemics) y la fonética (Pike, 1954). Mientras que la primera (emic) se ocupa de los fonemas, es decir, de los sonidos pertinentes (con sentido) para el hablante; la segunda (etic) estudia los sonidos emitidos por el hablante desde una perspectiva física y fisiológica sin tener en cuenta la opinión de quienes los efectúan. Pero mientras que la fonología y la fonética nos ofrecen productos de dispar naturaleza sin posible relación, respectivamente producen fonemas (es decir, unidades con sentido) y sonidos sin sentido, tanto la 
perspectiva emic como etic producen sentidos, y en consecuencia alguna relación se podrá establecer.

La dualidad emic/etic trasladada a las ciencias sociales es utilizada para distinguir entre el pensamiento que se tiene de las cosas (emic) y la cosas que se hacen (etic). Adjudicándose el enfoque emic a las opiniones de la población estudiada, mientras que el enfoque etic se asigna al que aplica el científico social al observar la conducta de los sujetos observados.

Marvin Harris sostiene que "tanto la dimensión emic como etic son tanto mentales como conductuales" (Harris, 1985, pp. 497-503). Este planteamiento supone un avance respecto a la distinción primigenia; sin embargo, se sigue manteniendo que la primera (emic) nos remite a la visión subjetiva del sujeto investigado; mientras que la segunda (etic) nos remite al proceder objetivo del sujeto investigador. Esta distinción no tiene en cuenta que la visión etic no es más que emic —esto es, la visión de la que parte el antropólogo profesional avalado por la comunidad académica- y la emic no es más que etic, dado que el antropólogo, a partir de la materia prima suministrada por la población nativa, es el que, desde su posición etic, da cuenta de la concepción que la población nativa tiene de la realidad sociocultural. No hay, pues, ningún estudio emic desde la perspectiva nativa. El análisis emic nativo es una quimera. Los trabajos de Carlos Castaneda (1993) serían los que más se aproximarían, pero no ha de olvidarse que las enseñanzas de Don Juan siempre son las enseñanzas que Castaneda dice que son las enseñanzas de Don Juan.

Por tanto, la cuestión a resolver no es si en la producción de conocimiento el investigador/científico social se ha de decantar por la perspectiva emic o etic, sino en propiciar que todas las perspectivas emic puedan ejercer de etic y, asimismo, que se asuma que todas las etic son emic; emic de un grupo sociocultural determinado. De tal suerte que todos suministren materia prima objeto de interpretación y toda interpretación sea materia prima objeto de interpretación.

\section{Descripción tecnológica}

Como se expone en el esquema siguiente (Figura 1), el desarrollo del proceso metodológico propuesto consta de seis momentos o fases que dan cuenta, a su vez, de otras tantas tareas específicas, aplicadas de manera flexible.

\section{Fase 1: Negociación inicial de la propuesta}

La realización de una investigación suele partir de un primer contacto entre una instancia demandante y una instancia técnica, entre un cliente (frecuentemente el financiador del trabajo) y un equipo técnico de investigación, pero en nuestro caso los conceptos clásicos se van difuminando y se complejizan. Los conceptos de demandante y cliente adquieren una dimensión diferente cuando se trata de un proceso participativo, en el que el cliente-demandante (es cliente para el equipo contratado) se convierte en participante y objeto de estudio y la población objeto de investigación e incluso el equipo técnico asumen el papel de participante-cliente y su trabajo es observado como parte de la investigación.

Desde la perspectiva sociopráxica este momento es determinante. Debe quedar claro que el informe de la investigación es un producto 

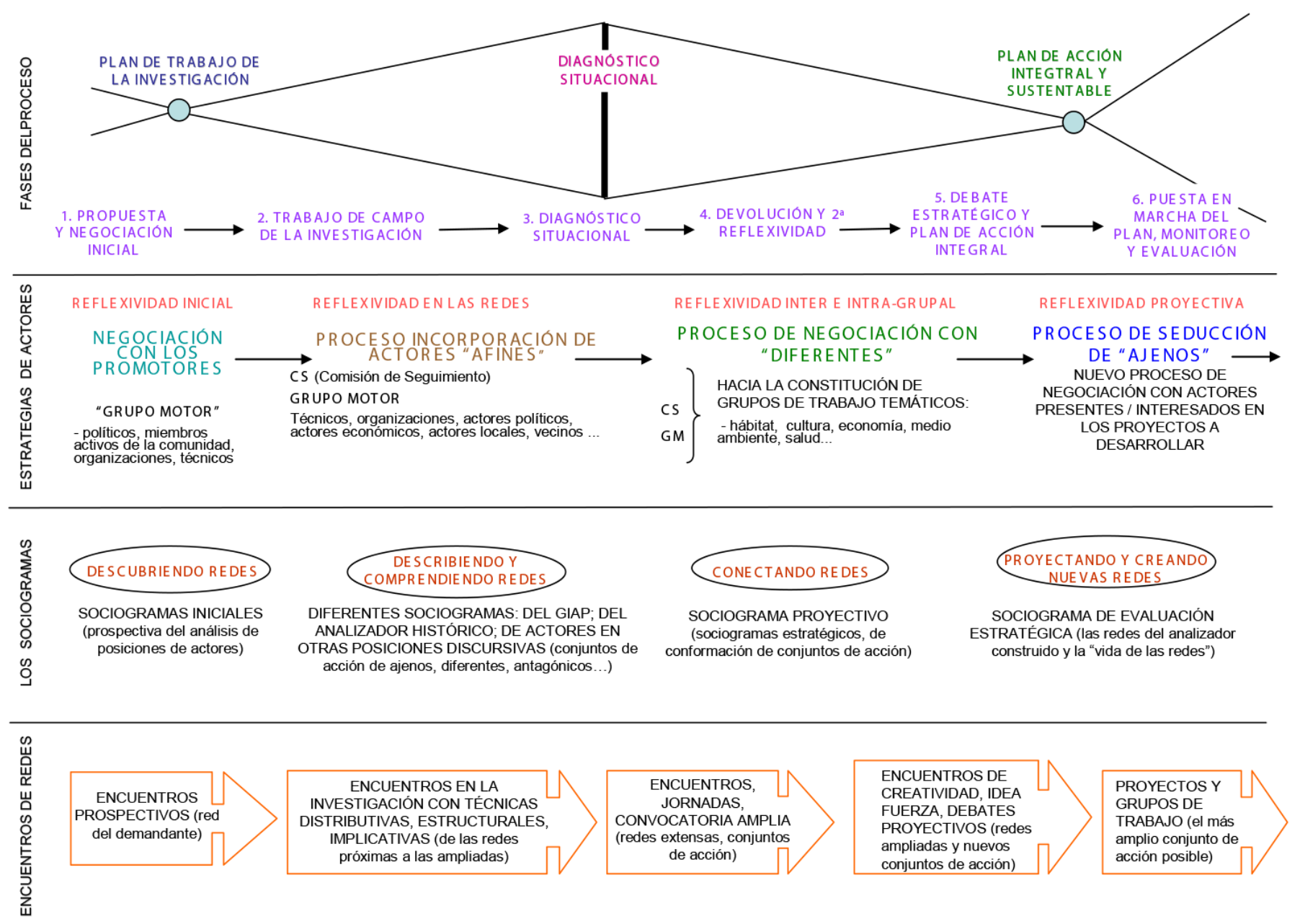

para el demandante, pero es preciso que toda la información circule, se comparta y socialice, como un imperativo epistemológico y metodológico de la investigación. Es un ejercicio de reflexión y autocrítica del equipo investigador sobre las posiciones comúnmente adoptadas en las investigaciones al uso, pero también ha de haber una autocrítica en el seno de las primeras conversaciones con los actores inicialmente dispuestos a participar.

Se considera adecuado que la elaboración del proyecto (delimitación del objeto de estudio, determinación de objetivos, sujetos inicialmente implicados, plan de trabajo, etc.) sea el fruto del debate con la mayor parte de actores a los que sea posible consultar inicialmente e implicar en la investigación. A tal fin, se ha de constituir un primer Grupo Motor (GM) que sea un grupo experto convivencial ${ }^{3}$ y que vaya informando al investigador en los saberes de la comunidad.

El grupo inicialmente constituido (demandantes, equipo técnico, primeros expertos consultados...) puede elaborar un primer sociograma e identificar a los actores de la red más cercana. De esta manera, se puede elaborar una primera negociación participada del proyecto de participación (permítasenos la redundancia). Pero no hemos de olvidar una cuestión clave: este grupo motor inicial se está moviendo en su red natural y una metodología participativa ha de plantearse la apertura a otros espacios, con el fin de no quedarse enredado en una sola red: en su red. La estrategia metodológica ha de ir de las redes más afines a las más antagónicas. También puede ser de utilidad el sociograma de

Figura 1. Resumen del Desarrollo del Método de Trabajo. Fuente: Elaboración propia. 
algún analizador histórico — como propone Fals Borda(1993)—, con el fin de hacer aflorar aquellos conflictos o alianzas que vienen de otros tiempos y otros acontecimientos.

También se ha de configurar una Comisión de Seguimiento (CS), cuya misión será la supervisión y orientación del proceso.

Para esta fase de inicio hemos de proponer algunas técnicas que son más pertinentes, por ejemplo, la matriz DAFO puede ayudarnos a hacer un somero diagnóstico que precise los aspectos más elementales relacionados con la problemática de investigación. De cara a la segunda fase, se ha de diseñar un plan de comunicación que informe del proceso iniciado a otros actores que aún no sean conocedores del mismo.

\section{Fase 2: Trabajo en las redes}

En esta fase lo conveniente es generar el conocimiento indispensable sobre la problemática objeto de investigación, para que pueda ser debatido, reflexionado y así obtener propuestas que afronten (de manera participada) la problemática que inicialmente se ha construido. Para ello hemos de echar mano de los métodos y técnicas más apropiadas para investigar el tema, recurriendo a una lógica plural, abierta e integradora y a las distintas triangulaciones de disciplinas científicas, de métodos y técnicas de investigación. La estrategia del diseño del trabajo a realizar en campo ha de responder al diseño de una muestra. Si bien no queremos construir un sistema que informe del universo de manera programada (informando solo de aquello de lo que puede dar cuenta porque está controlado desde fuera), sino construyendo un dispositivo que pueda generar su propia información, así como la manera de articularla, lo que proponemos es elaborar una muestra que se base en la estructura de las redes de acuerdo con los sociogramas elaborados al efecto.

En cuanto a las técnicas a emplear, el criterio por el que hemos optado ha sido el de pertinencia en relación con el propósito que se persigue (la finalidad y los objetivos de la investigación y planificación), además de la situación concreta en que nos encontramos. Proponemos "seis tipos de técnicas con las que podemos proceder, agrupadas en estas categorías: dinamizadoras, documentales, distributivas, estructurales, implicativas/reflexivas y conversacionales" (Montañés, 2009, p. 121).

Estas categorías proponemos cruzarlas con los siguientes principios de utilización de las técnicas, al objeto de encontrar la adecuación pertinente a su cometido:

- dependiendo de la aplicación a los objetivos;

- dependiendo de las características de los sujetos;

- dependiendo de los recursos;

- dependiendo de la experiencia en su manejo por los participantes;

- dependiendo de la transferencia de tecnología social y de la tecnología apropiada de la técnica en sí.

4 Hemos advertido sobre el error de suponer que la participación implica convertir a quienes la practican en economistas, biólogos, abogados o sociólogos; pero es igual de erróneo el que, para participar, cada actor deban despojarse de sus conocimientos.
El primero de los criterios ha de tener un peso diferente por su adecuación al proyecto de investigación, en concreto a sus objetivos y finalidad, porque el resto son criterios de carácter más ambiental o situacional, en función de los participantes. Pero quede también claro que esto no significa que el equipo de investigación deba abjurar de su trabajo, que se le exima de hacer lo que le es propio, el manejar el método y las técnicas y llevar la responsabilidad del desarrollo de la investigación. ${ }^{4}$ 
Todo lo contrario, los técnicos han de manejar información (y también teoría) y técnicas, pero para ponerlas al servicio del aprendizaje reflexivo de todos los participantes.

\section{Fase 3: Diagnóstico (apreciación) situacional}

Los diagnósticos que se realizan dependen siempre de las preguntas que se enuncian. No conforman reflejos exactos de la realidad, sino - en el mejor de los casos- son como fotografías parciales y provisionales con una multiplicidad de ángulos de visión y de intereses de los distintos observadores. Esta diversidad puede percibirse, a primera vista, contradictoria, incoherente y paradójica, como un conjunto coral polifónico y disonante al mismo tiempo. El que las paradojas nos desconcierten, pero nos den pistas sobre cómo funciona la realidad que nos ocupa, es una estrategia nómada de persecución itinerante de las dimensiones de explicación. Esto requiere, por tanto, de la reflexividad de los diversos actores.

Toda esta información que se va produciendo por los diferentes grupos o personas participantes en el proceso ha de ser interpretada, lo que no equivale a que deba ser el equipo técnico de investigación quien únicamente interprete la materia discursiva que se produce. Pero el grupo de investigación tampoco ha de eludir la tarea que le es propia y ha de proceder a interpretar los discursos, con los procedimientos propios del método de investigación, de manera que se agregue a la interpretación de los protagonistas; esta es la complementación de la actividad de los expertos convivenciales y la de los metodológicos y temáticos.

\section{Fase 4: Dialógica informativa y segunda reflexividad}

Desde una perspectiva de investigación sociopráxica, lo que se denomina comúnmente como diagnóstico es fundamentalmente el punto de arranque del autodiagnóstico de los actores sociales participantes. Una tarea clave de los investigadores consiste en encontrar los medios para traducir la jerga de los datos técnicos al lenguaje y la comprensión de los participantes, pero no para convencer de los resultados de sus indagaciones, sino para problematizar el conocimiento de unos y otros; así, el diagnóstico se convierte en una herramienta de reflexión y educación mutuas.

Una manera de problematizar la información del diagnóstico es mostrar los dilemas en que suelen encajonarse, pero complejizados mediante cuadros sémicos, para saltar del espacio cristalizado en dos categorías a otros emergentes y complejos, que posibiliten las acciones más creativas y emancipadoras.

En muchas ocasiones el diagnóstico de la situación puede ser excesivamente minucioso o bien pecar de superficialidad, con el fin de avanzar hacia la fase de planificación, de acuerdo con una simple estrategia pragmática de comunicación (además del rigor que ha de tener), conviene priorizar los problemas más importantes, su articulación, las causas fundamentales sobre las que se deberá actuar, pues solo así se podrá ir hacia un plan de acción realista y viable.

Conviene subrayar que en el contexto histórico presente, la participación no surgirá por la invitación bienintencionada a participar; es preciso pensar en otras modalidades de comunicación y estímulo. Por ejemplo, planteándose la validez y eficacia de los más variados ins- 
trumentos, desde las técnicas escénicas a las audiovisuales, las nuevas tecnologías de la información y la comunicación o las más convencionales, presentadas en escenarios de encuentro y convivencia o en reuniones convocadas al efecto.

\section{Fase 5: Debate estratégico y articulación de propuestas}

Esta fase se enlaza con el debate reflexivo del diagnóstico, con la apropiación por los participantes de los resultados obtenidos, propiciando la elaboración de un Plan de Acción surgido de las propuestas hechas por los diferentes actores. El uso en los encuentros de creatividad social de técnicas como el flujograma ha dado resultados más satisfactorios que los de la matriz DAFO, que consideramos más ambigua y rígida para estos momentos de la planificación. Las relaciones múltiples y recursivas entre causas y efectos complejizan cada uno de los $n u$ dos críticos de los problemas a abordar, si bien, esos nudos críticos han de ser debatidos también de manera creativa.

Planificar es imaginar un escenario deseado de futuro y poner los medios, la organización y los recursos para construirlo tal como se ha soñado. Por consiguiente, se ha de debatir con los participantes sobre cómo desean construir su futuro. Para realizar este plan de acción es preciso plantearse previamente su sentido y este es un debate estratégico, tal como lo plantea Morin: apertura de las programaciones frente al plan cerrado, flexibilidad y control del proceso frente a la rigidez de la evaluación de impacto, la estrategia con la que afrontar la incertidumbre frente a la certeza y la determinación de la planificación normativa.

"Una estrategia se determina teniendo en cuenta una situación aleatoria, elementos adversos e, inclusive, adversarios y está destinada a modificarse en función de las informaciones provistas durante el proceso" (Morin, 1994, p. 127).

Por tanto, y para resumir, en esta fase se ha de plantear un debate estratégico sobre la toma de decisiones en torno a qué idea fuerza es la que daría sentido al proceso, con las acciones que aborden el problema de manera integral (desde los distintos temas que lo compongan, en la realidad indivisible de la comunidad) y con la formación del más amplio conjunto de acción entre los actores participantes y que están dispuestos a apoyar las ideas-fuerza; a esto lo llamamos Plan de Acción Integral y Sostenible (PAIS).

\section{Fase 6: Realización del plan, monitoreo y evaluación}

La puesta en práctica del PAIS es fundamental dentro de una metodología que tenga entre sus pilares el de construir conocimiento, el de aprender. Por lo tanto, si en esta fase se considera que ya no es precisa la participación, se está hurtando a los actores intervinientes la posibilidad de comprobar si aquello que se llevaría a cabo era o no lo que se había decidido, de aprender a bregar con la incertidumbre en la tarea de hacer posible lo que se ha decidido.

La continuidad de la participación en la puesta en marcha del Plan viene acompañada de dos tareas que no hay que confundir: el seguimiento (monitorización) y la evaluación. Aunque podemos encuadrar ambos aspectos como dos caras del mismo mecanismo, sin embargo, tienen diferentes intenciones y aportan distintos frutos. El seguimiento o monitorización ha de aportar la información para la toma de decisiones y lo puede realizar quienes midan, con los indicadores apropiados 
y acordados, cómo se va desarrollando el Plan. Hablar de evaluación supone, en términos operativos, hacer un juicio de valor de los resultados que va logrando el Plan en su proceso, y, por tanto, no quiere decir que tenga que hacerse solo al finalizar este. Supone una toma democrática de decisiones, que en este tipo de procesos ha de hacerse en aquel espacio organizativo acordado. Planificar y evaluar son dos procesos que han de caminar de la mano. La realización de una evaluación de tipo participativo sería en parte un tema diferente y en parte otra aplicación del mismo proceso.

Recapitulando, es necesario que, acompañando al PAIS, se elaborase un plan de evaluación, que plantee con todo detalle la organización, los momentos y ámbitos de aplicación, etc., y todo ello en términos participativos.

\section{Reflexiones finales}

Las prácticas, enmarcadas en un proceso reflexivo transductivo, han sido las fuentes de las que se nutre la propuesta formulada. Se ha elaborado una síntesis de procedimientos metodológicos a partir de la reconsideración de los principios epistemológicos de la IAP y las diferentes metodologías participativas y práctica diversas, buena parte de ellas procedentes de América latina, como recoge una obra colectiva de reciente aparición en la que participan los autores de este artículo (Red CIMAS, 2015). En este sentido, la intención, en los más de tres lustros a los que se ha hecho referencia, ha sido la de desbordar de manera crítica y creativa a lo ya ensayado, pues como afirmaba Fals Borda:

Tenemos que mirar más allá de la IAP, porque la actual etapa de la cooptación nos llevará a expresiones cualitativas muy diferentes que ojalá conserven los primeros objetivos de aquélla. No sabemos todavía en lo que resultará: quizás en una investigación participativa enriquecida y más creadora (Fals Borda, 1993, p. 19).

A lo que nos hemos resistido, en cada caso, con mejor o peor fortuna, ha sido a renunciar a la vertiente política de la práctica investigadora participativa, entendido este aspecto como la intervención activa de la población en lo que le es propio, para decidir cómo organizar su vida en comunidad, hacia situaciones más igualitarias, dignas y justas como así nos lo han demandado explícitamente, en no pocas ocasiones, las personas y organizaciones con quienes hemos trabajado.

En el mismo sentido hemos perseguido que los procesos de investigación-acción-reflexión produzcan conocimiento, no solo para los investigadores (equipo de expertos metodológicos) o para los demandantes-financiadores, sino para toda persona y grupo participante, con quienes nos educamos (mutuamente) en la transformación de la realidad. De este modo, todos y todas somos educadas y nos transformamos, pues, como se ha dicho al principio, en los procesos sociopráxicos de transformación social todas y todos nos transformamos en la acción de transformar. 


\section{Referencias}

Briones, G. (1978). Sobre cuestiones de objeto y métodos en la investigación militante. En Molano, A. (comp.), Crítica y política en ciencias sociales. Bogotá: Punta de Lanza.

Castaneda, C. (1993). Las enseñanzas de Don Juan. Madrid: FCE.

Crespo, E. (2003). El construccionismo y la cognición social: metáforas de la muerte. Política y Sociedad, 40 (1), 15-26.

Delorme, C. (1982). De la animación pedagógica a la Investigación-Acción. Madrid: Nercea.

Desroche, H. (1979). La recherche et l'action: Vers une 'nouvelle sociologie'. Communaut's, 48.

Descartes, R. (1961). Discurso del método. Buenos Aires: Losada.

Faber, B.A. et al. (2001). La psicoterapia de Carl Rogers. Bilbao: Desclée de Brouwer.

Fals Borda, O. (1993). La investigación participativa y la intervención social. Documentación Social, 92, 9-21.

Freire, P. (1970). Pedagogía del oprimido. Madrid: Siglo XXI.

Galeano, E. (1998). Patas arribas. La escuela del mundo al revés. Madrid: Siglo XXI.

Ganuza, E. y Francés, F. (2012). El círculo virtuoso de la democracia: los presupuestos participativos a debate. Madrid: CIS

Greenwood, D. (1998). Investigación-acción y desarrollo de una comunidad en una ciudad de La Mancha. Perspectivas de Gestión, III (1), 28-36.

Greenwood, D. y González, J.L. (1989). Culturas de Fagor. Estudio antropológico de las cooperativas de Fagor. Donosti: Txertoa.

Harris, M. (1985). El desarrollo de la teoría antropológica. Historia de las Teorias de la Cultura. Madrid: Siglo XXI.

Ibáñez, J. (1986). Más allá de la sociología. El grupo de discusión. Madrid: Siglo XXI.

- (1990). Introducción a los nuevos avances de la investigación social. La investigación de segundo orden. Anthropos, 22, suplemento.

Kemmis, S. y McTaggart, R. (1987). Cómo planificar la investigación acción. Barcelona: Laertes.

Lewin, K. (1946). Action research and minority problems. Journal of Social Issues, 2.

López de Ceballos, P. (1987). Un método para la investigación-acción participativa. Madrid: Editorial Popular.

Martín Gutiérrez, P. (2010). La planificación participativa desde una perspectiva de redes sociales. Tesis doctoral. Madrid: UCM.

Maturana, H. y Varela, F. (1990). El árbol del conocimiento. Madrid: Debate.

Meister, A. y Retour, D. (1980). Pérou 1968-1980: matériaux l'expérience autogestionnaire. Autogestions, 4.
Molano, A. (1978). Crítica y política en ciencias sociales. Bogotá: Punta de Lanza.

Montañés, M. (2003). El diseño del proyecto de investigación/planificación social participada. En Praxis Participativas desde el Medio Rural. Madrid: Iepala Editorial.

- (2009). Metodología y Técnica participativa (Teoría y práctica de una estrategia de investigación participativa). Barcelona: Editorial UOC.

Montañés, M. et al. (1999). Estudio de necesidades de formación de los Agentes de Desarrollo Local y las figuras profesionales afines. Madrid: FOREM-CC.OO.

Montes del Castillo, A. (1989). Simbolismo y Poder. Un estudio antropológico sobre compadrazgo y priostazgo en una comunidad andina. Barcelona: Anthropos.

- (1993). Investigación Acción en Antropología Social. En Documentación Social, 93, 177-188.

Morin, E. (1995). Introducción al pensamiento complejo. Barcelona: Gedisa.

- (1994). La noción de sujeto y epistemología de la complejidad. En Fried Schnitman, Dora (comp.), Nuevos paradigmas, cultura y subjetividad. Barcelona: Paidós.

Núñez, C. et al. (1990). Investigación Participativa y Educación Popular en América latina. México: IMDC, AC.

Oquist, P. (1978). Epistemología de la investigación-acción. En Molano, A. (comp.), Crítica y política en ciencias sociales. Bogotá: Punta de Lanza.

Pask, G. (1981). Organizational Closure of Potentially Conscious Systems. En Zeleny, M. (ed.), Autopoiesis. A theory of the living organization. North Holland.

Pike, K.L. (1954). Language in relation to a unified theory of the structure of human behaviour. Mouton, 1.

Red CIMAS (2015). Metodologías participativas. Sociopraxis para la creatividad social. Madrid: Dextra.

Rojas, F. (1978). El apego de las ciencias sociales a lo real. En Molano, A. (comp.), Crítica y política en ciencias sociales. Bogotá: Punta de Lanza.

Sanguinetti, Y. (1981). La investigación participativa en los procesos de desarrollo de América latina. Revista de la Asociación Latinoamericana de Psicología Social.

Schutter, A. de (1985). La investigación-acción. México: GREFA.

UCM y Ayuntamiento de Fuenlabrada (1995). Convenio de colaboración UCM Ayuntamiento de Fuenlabrada. Madrid: S/E.

Vio Grossi, F. (1981). Investigación participativa: precisiones de Ayacucho. En VVAA, Investigación participativa y praxis rural. Lima: Mosca Azul.

Von Glasersfeld, E. (1994). La construcción del conocimiento. En Fried Schnitman, Dora (comp.), Nuevos paradigmas, cultura y subjetividad. Buenos Aires: Paidós. 
MoMontañés Serrano, M. y Martín Gutiérrez, P. (2017). De la IAP a las Metodologías Sociopráxicas. Hábitat y Sociedad, 10, 3552.

<http://dx.doi.org/10.12795/HabitatySociedad.2017.i10.03>

$\bigcirc$ 


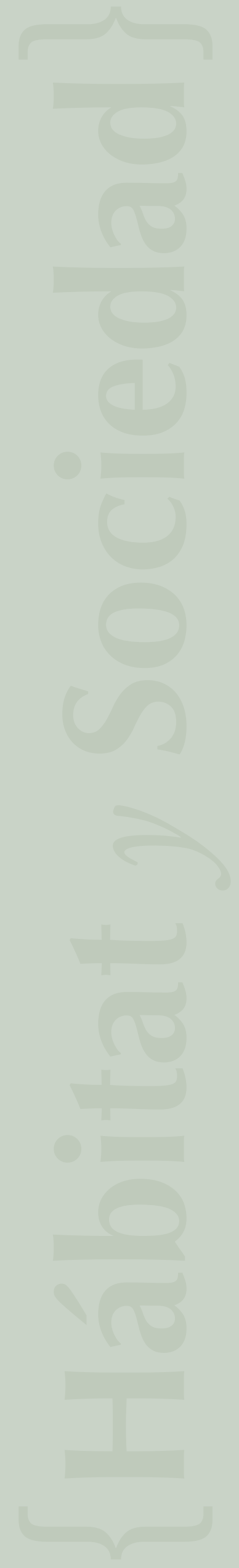

